Highlighting work from the Böker Research Group, Fraunhofer Institute for Applied Polymer Research (IAP), Potsdam, Germany.

Measuring rotational diffusion of colloidal spheres with confocal microscopy

A confocal-microscopy-based method is reported to concurrently measure the translational and rotational dynamics of colloidal spheres in three dimensions.

\section{As featured in:}

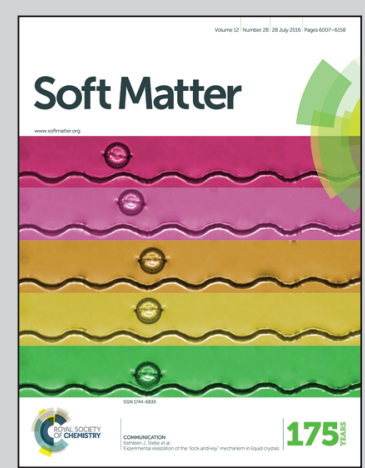

See Bing Liu and Alexander Böker, Soft Matter, 2016, 12, 6033. 


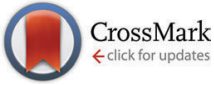

Cite this: Soft Matter, 2016, 12,6033

Received 9th May 2016 Accepted 14th June 2016

DOI: 10.1039/c6sm01082b

www.rsc.org/softmatter

\title{
Measuring rotational diffusion of colloidal spheres with confocal microscopy $\dagger$
}

\author{
Bing Liu*abc and Alexander Böker ${ }^{\text {abd }}$
}

\begin{abstract}
We report an experimental method to measure the translational and rotational dynamics of colloidal spheres in three dimensions with confocal microscopy and show that the experimental values reasonably agree with the theoretical values. This method can be extended to study rotational dynamics in concentrated colloidal systems and complex bio-systems.
\end{abstract}

Measuring the diffusion of colloidal particles in a complex fluid is central for understanding the dynamic behavior of colloids. ${ }^{1}$ The aging behavior of glassy colloidal systems, the enhanced motion of active colloids, and molecular motors in biological processes are typical examples. ${ }^{2-4}$ Additionally, colloidal diffusion is reported to be used for exploring the microrheology of soft materials. ${ }^{5,6}$ The translational diffusion of colloidal spheres is relatively accessible and has been extensively studied. Rotational diffusion, however, is much more difficult to study due to the spherical symmetry of the particles. Several methods to measure the rotational diffusion of colloidal spheres have been reported, including dynamic depolarized light scattering (DDLS), ${ }^{7}$ dynamic evanescent wave scattering, ${ }^{8}$ deuteron NMR, ${ }^{9}$ and polarized fluorescence recovery after photobleaching (pFRAP).$^{10}$ Structural and dynamic heterogeneities are typically difficult to detect by such ensemble-averaging methods. Also, these methods are not suitable for systems in complex biological environments. ${ }^{11}$

In recent years, digital video microscopy is attracting much attention as a powerful tool for the direct visualization of colloidal suspensions at a single particle level. Together with

\footnotetext{
${ }^{a}$ Fraunhofer Institut für angewandte Polymerforschung (IAP), Geiselbergstr. 69, 14476 Potsdam, Germany. E-mail: liubing@iccas.ac.cn

${ }^{b} D W I$ - Leibniz Institute for Interactive Materials e.V., Lehrstuhl für Makromolekulare Materialien und Oberflächen, RWTH Aachen University, Forckenbeckstr. 50, D-52056 Aachen, Germany ${ }^{c}$ State Key Laboratory of Polymer Physics and Chemistry, Institute of Chemistry, Chinese Academy of Sciences, 100190, Beijing, China

${ }^{d}$ Lehrstuhl für Polymermaterialien und Polymertechnologien, Universität Potsdam, Geiselbergstrasse 69, 14476 Potsdam, Germany

$\dagger$ Electronic supplementary information (ESI) available: Details of the synthesis of model colloids and supporting figures. See DOI: 10.1039/c6sm01082b
}

advanced computer tracking algorithms, digital video microscopy offers sub-pixel resolution for the identification of the positions of colloidal particles. ${ }^{12}$ This method is extensively used to track the translational diffusion of both spherical colloids and anisotropic colloids. ${ }^{12-14}$ Especially for the latter, for examples rods or ellipsoids, this method can also be extended to measure the rotational diffusion by tracking any identifiable optical axis of each particle. ${ }^{13,14}$ However, this straightforward strategy is not applicable for isotropic spherical colloids due to the lack of such identifiable optical axes. While retaining the advantages of single particle tracking, digital holography microscopy has shown the ability to measure the rotational dynamics of a single particle by recording a $2 \mathrm{D}$ holographic interference pattern and then fitting it with appropriate theoretical models. Examples of the measured colloidal particles reported include rods, dumbbells and spheres. ${ }^{15,16}$ The drawback of this method is its expensive computational process. Modulated optical probes (MOONs) provide another effective way to track colloidal rotation at a single particle level. The probe consists of a fluorescent spherical particle that is half coated with metal. The metal cap can modulate the excitation and emission of fluorescence, resulting in moon-like patterns, thus enabling the tracking of rotation. ${ }^{17,18}$ Unfortunately, this method is more suitable for the study of a colloidal system in two dimensions.

Traditional bright field microscopy is limited due to its low resolution in the $z$ axis. With a confocal pinhole, confocal microscopy can precisely resolve the positions of colloidal particles in three dimensions. ${ }^{19}$ As such, confocal microscopy has been widely used for real space imaging of colloidal systems in colloidal physics, concerning a variety of topics such as crystallization, melting and freezing, and glass transition. ${ }^{20-24}$ Some groups have developed methods based on confocal microscopy to measure the rotation of anisotropic particles. ${ }^{13,14}$ However, for colloidal spheres, their rotational dynamics have not been greatly explored with confocal microscopy to date. Rotational diffusion of colloidal spheres has been used to explore the decoupling of translational-rotational motion in concentrated colloidal systems. ${ }^{25,26}$ In this communication, we demonstrate a 

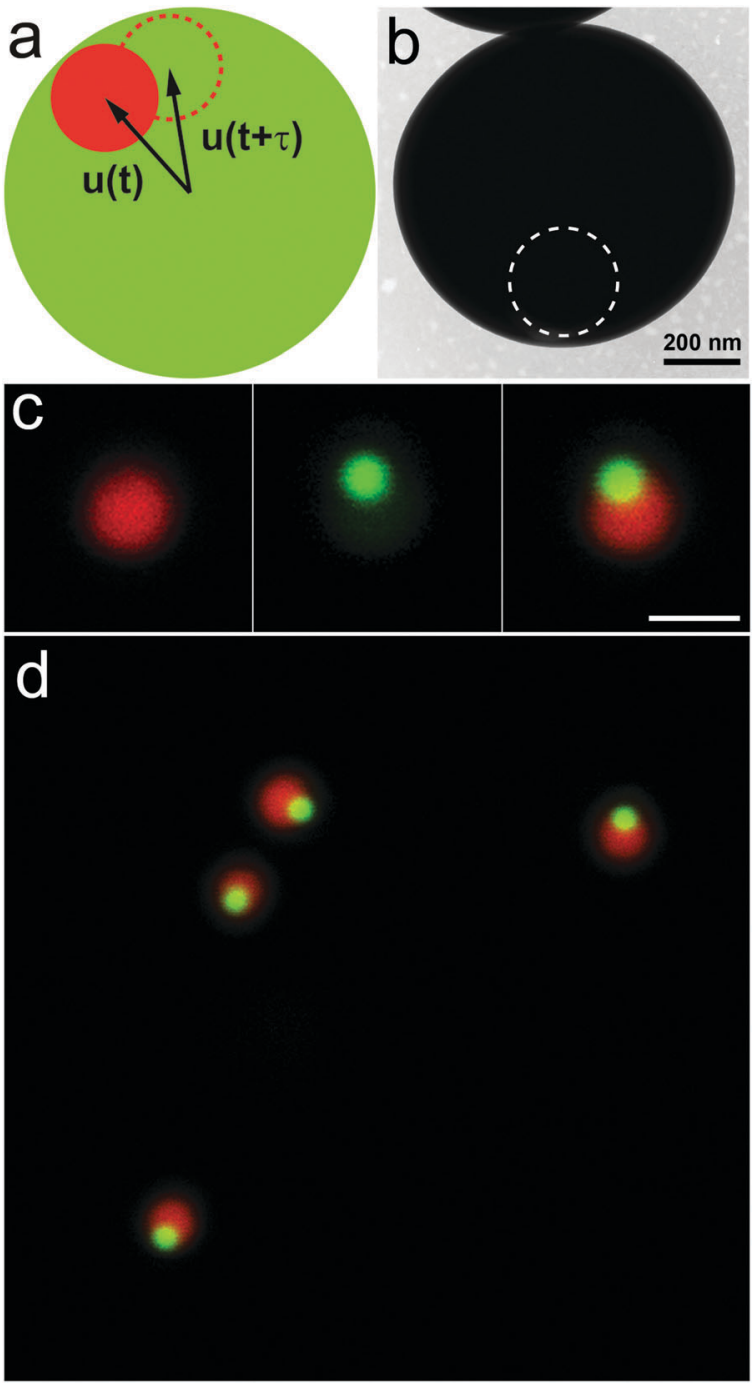

Fig. 1 Illustration of the method of measuring rotational diffusion using eccentric colloidal spheres. (a) Schematic model. $\mathbf{u}(t)$ is the unit vector from the center of the shell pointing to the center of the core. (b) TEM image of an eccentric particle. The dashed circle indicates the position of the small particle. (c) Single channels of an eccentric particle showing two distinct colors excited by two different laser lines, $458 \mathrm{~nm}$ (left) and $561 \mathrm{~nm}$ (middle). The red color indicates the position of the shell and the green color indicates the position of the core. The right image in (c) shows an overlay of the two channels. (d) A confocal image overview showing several of the particles shown in (c). The scale bar in (c) is $1 \mu \mathrm{m}$.

quantitative method to concurrently measure the translational and rotational diffusion of colloidal spheres with confocal microscopy. Our strategy is to build an identifiable fluorescent optical axis inside colloidal spheres via chemical synthesis while retaining the uniform surface chemistry of each colloidal sphere. To realize this idea, we devised and synthesized eccentric coreshell colloids (Fig. 1a) where the core was marked with one dye and the shell was marked with another dye. The two dyes are readily distinguishable with confocal microscopy; thus, the center of mass of either the core or the shell can be identified independently. The optical axis is referred to the vector from the center of the shell to the center of the core, as shown in Fig. 1a.
By following the motion of this optical axis with a confocal microscope, we can determine the rotational dynamics. In this communication, we validate this method for colloidal spheres in dilute suspensions as a proof-of-principle.

Monodisperse fluorescent colloidal silica spheres with a diameter of $247 \mathrm{~nm}$ (measured by TEM, polydispersity $4.3 \%$ : mean value divided by standard deviation) was synthesized as described in ref. 27. The dye molecule rhodamine B isothiocyanate (RITC) was covalently incorporated into the silica spheres to enable visualization under a confocal microscope. The silica spheres were then modified with 3-methacryloxypropyl trimethoxysilane (MPTS) and then grafted with a thin layer (ca. $20 \mathrm{~nm}$ ) of polystyrene (PS) via emulsion polymerization. Finally, we obtained eccentric core-shell colloidal spheres, denoted as $\mathrm{SiO}_{2} /$ poly(MPTS), according to the reported procedure in ref. 28 (Fig. $1 \mathrm{~b}$ and Fig. S1, ESI $\dagger$ ). The core is $\mathrm{SiO}_{2}$ and the shell is the polymerized MPTS. The as-prepared particles have a diameter of $941 \mathrm{~nm}$ (measured by TEM, polydispersity $5.7 \%$ : mean value divided by standard deviation). The poly(MPTS) shell was marked with the dye coumarin 153.

The $\mathrm{SiO}_{2} /$ poly(MPTS) colloidal spheres were dispersed into solvents with very similar refractive indices. We tested two systems: one is a mixture of dimethyl sulfoxide (DMSO) and glycerol $(1: 3.53, \mathrm{~m} / \mathrm{m})\left(n_{\mathrm{e}, \mathrm{mix}}=1.4750, \eta=0.23 \mathrm{~Pa} \mathrm{~s}\right)$ and the other is Triton $\mathrm{X}-100\left(n_{\mathrm{e}}=1.4920, \eta=0.33 \mathrm{~Pa} \mathrm{~s}\right)$. All the measurements were performed with a volume fraction less than 0.005 so that the colloidal spheres formed an extremely dilute suspension where they did not interact with each other. The samples were studied with a laser scanning confocal microscope (Leica TCS SP8 equipped with a $12000 \mathrm{kHz}$ resonant scanner). All images were acquired in fluorescence mode. Two excitation wavelengths, $458 \mathrm{~nm}$ and $561 \mathrm{~nm}$, were used for coumarin 153 and RITC, respectively. The scanning time of each series was $1.03 \mathrm{~s}$, achieved by scanning $512 \times 256 \times 67$ pixels with a pixel size of $24 \mathrm{~nm}$ in $x-y$ and $150 \mathrm{~nm}$ in $z$. Typically, the data recording requires 500 repeats to achieve reliable statistics. We extracted the positional coordinates of the core and the shell using a standard Interactive Data Language (IDL) route ${ }^{12}$ and calculated the vector of the optical axis via a home-made program. In order to successfully identify the centers of mass of the core and the shell, it was necessary for us to use two highly distinguishable dyes in a single colloid. As mentioned above, we used RITC for the core and coumarin 153 for the shell. In the free state, RITC typically has excitation and emission peaks at $546 \mathrm{~nm}$ and $568 \mathrm{~nm}$ and coumarin 153 typically has excitation and emission peaks at $422 \mathrm{~nm}$ and $532 \mathrm{~nm}$ in ethanol, respectively. We used two different laser lines to excite the dyes separately, and the fluorescence signals were collected by two detector channels. To further avoid crosstalk between the two dyes, we used sequential scanning mode. In this case, either the shell or the core can be imaged independently; therefore, we can also identify their centers of brightness independently (Fig. 1c and d). For the core, the center of brightness is the center of mass, and for the shell, the center of brightness has a slight deviation $(<10 \mathrm{~nm})$ from the geometrical center. In this case, the vector of the optical axis of each particle can thus be uniquely tracked, which makes it 
possible to track those angular displacements to $180^{\circ}$. However, for colloidal particles with symmetries, such as rods, an angular displacement of less than $90^{\circ}$ is normally required, as the two ends are not distinguishable. ${ }^{13,14}$ The trajectories of the center of mass and the orientations of the spheres were obtained by particle tracking.

The trajectories of a single particle for the free translational and rotational motions are shown in Fig. 2a and b, respectively, spanning a time period of $515 \mathrm{~s}$ (equal to 500 stacks). Sedimentation induced by the density mismatch between the $\mathrm{SiO}_{2} /$ poly(MPTS) colloids and the solvent does not show an obvious influence during this measured time period. Following the method suggested by Savin and Doyle ${ }^{29}$ we evaluated the static error in determining the coordinates of the rotational optical axis. The colloidal particles were immobilized into a similar sample cell and imaging was performed under the same conditions as the other experiments. The static error was found to be $5.5^{\circ}$ in our measurements. For a motion-free colloidal sphere, its translational and rotational diffusions in a viscous solvent are expected to appear Gaussian. Since our method allows us to track the diffusion of each colloidal sphere for all five degrees of freedom, we plotted the probability distribution of both the center of mass translational displacements and the optical axis vector angular displacements in Fig. 3. We typically measured 5 to 15 particles under the same conditions for statistical purposes. Indeed, as shown by the fitting curves
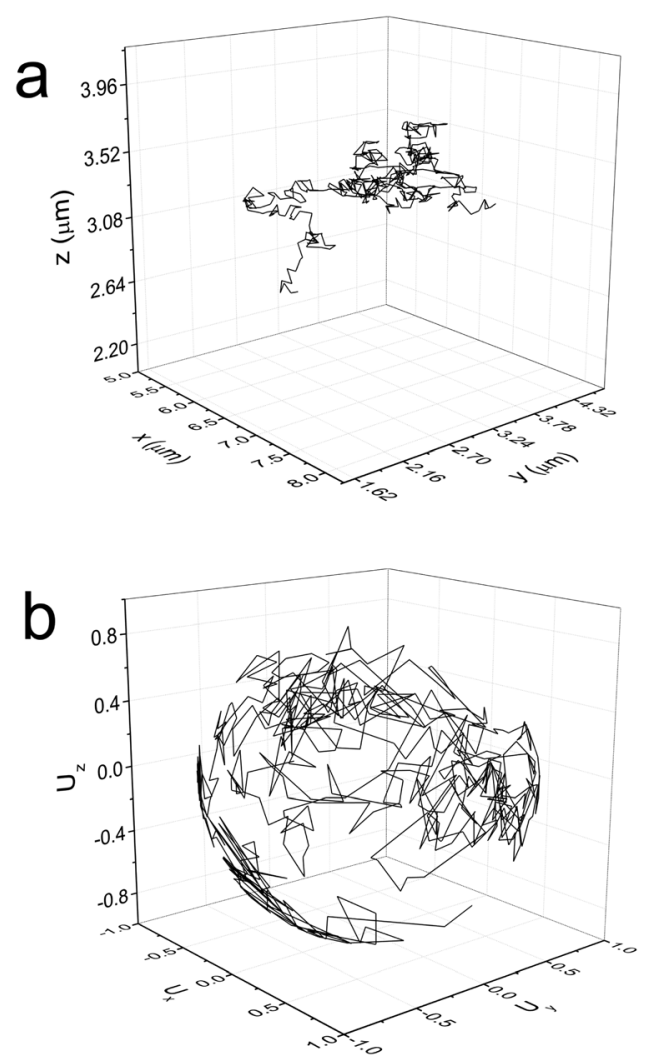

Fig. 2 (a) Translational and (b) rotational trajectories of a single particle. The trajectories span a time period of $515 \mathrm{~s}$. The dispersion media is a mixture of glycerol and DMSO.
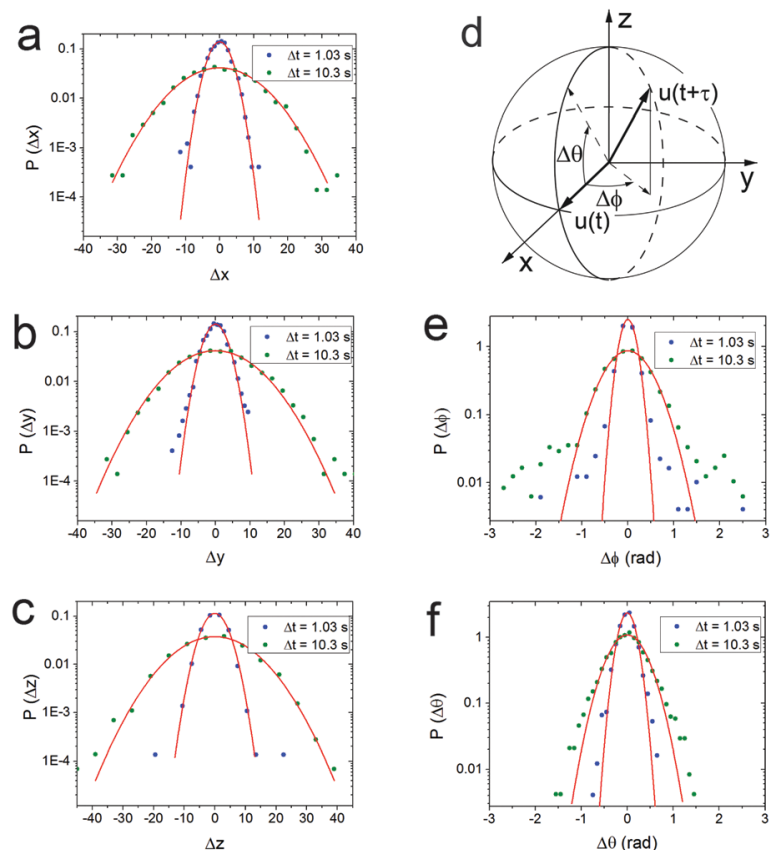

Fig. 3 The probability distribution of particle translational $(a-c)$ and rotational (e and f) displacements. Each plot was fitted with a single Gaussian function (red curves). The blue dots are for $\Delta t=1.03 \mathrm{~s}$ and the green dots are for $\Delta t=$ $10.30 \mathrm{~s}$. The dispersion media is a mixture of glycerol and DMSO. A schematic of one coordinate system for colloidal sphere rotation is shown in (d).

(Fig. 3 and Fig. S2, ESI $\dagger$ ), they are approximated by Gaussian functions. We also noticed a deviation from Gaussian distribution at large angular displacements for two rotational degrees of freedom; this could originate from the limited statistics, as we found that the large displacement in $\Delta \phi$ is mainly induced by a small angular displacement in $\mathbf{u}(t)-\mathbf{u}(0)$ of the optical axis around the polar axis. It should be mentioned that the density mismatch between the silica core and the poly(MPTS) shell also induced a deviation of the geometric center from the center of mass, and this deviation is less than $10 \mathrm{~nm}$ in our case by a simplified estimation. This effect should be negligible. We calculated the mean square displacement and mean angular square displacement from these trajectories, and the results are summarized in Fig. 4. To obtain the translational diffusion coefficient, we employed the formula

$$
\overline{\Delta \mathbf{r}^{2}}=6 D_{\mathrm{t}}\left(t-\frac{\tau}{3}\right)+6 \varepsilon^{2}
$$

where $D_{\mathrm{t}}$ is the translational diffusion coefficient, $\tau$ is the image acquisition time and $\varepsilon$ is the error. By fitting the translational dynamics to this formula, we obtained a translational diffusion coefficient $D_{\mathrm{t}}=2.60 \pm 0.04 \times 10^{-3} \mu \mathrm{m}^{2} \mathrm{~s}^{-1}$ with an estimated error $\varepsilon \approx 40 \mathrm{~nm}$.

To obtain the rotational diffusion coefficient, we employed the formula

$$
\overline{\Delta \mathbf{u}^{2}(t)}=2\left(1-\mathrm{e}^{-2 D_{\mathrm{r}} t}\right)+2 \varepsilon^{2}
$$

where $D_{\mathrm{r}}$ is the rotational diffusion coefficient and $\varepsilon$ is the error. By fitting the rotational dynamics to eqn (2), we obtained a 

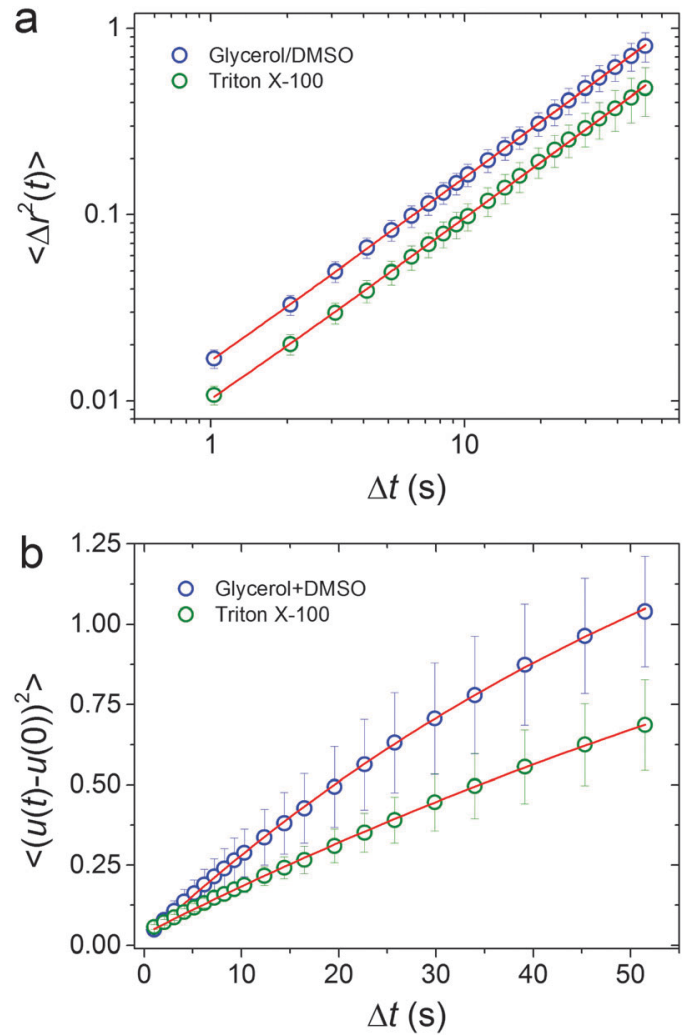

Fig. 4 The mean square displacements (a) and the mean angular square displacements (b) of eccentric core-shell $\mathrm{SiO}_{2} /$ poly(MPTS) colloidal spheres. Two dispersing media were used: a mixture of glycerol and DMSO, and Triton X-100. The circles are data, calculated from the measurements, and the solid curve is the fit with formula (1) for translational motion and formula (2) for rotational motion. The mass ratio of glycerol to DMSO is 3.53.

rotational diffusional coefficient $D_{\mathrm{r}}=7.04 \pm 0.18 \times 10^{-3} \mathrm{rad}^{2} \mathrm{~s}^{-1}$ with an estimated error $\varepsilon \approx 5.5^{\circ}$.

As a second test, we used Triton X-100 as the solvent instead of the mixture of DMSO and glycerol. Following a similar procedure, we obtained a translational diffusion coefficient $D_{\mathrm{t}}=1.56 \pm 0.06 \times 10^{-3} \mu \mathrm{m}^{2} \mathrm{~s}^{-1}$ with an estimated error $\varepsilon \approx 40 \mathrm{~nm}$ and a rotational diffusion coefficient $D_{\mathrm{r}}=3.83 \pm$ $0.08 \times 10^{-3} \operatorname{rad}^{2} \mathrm{~s}^{-1}$ with an estimated error $\varepsilon \approx 7.7^{\circ}$. A comparison between the measured values and the theoretical values is summarized in Table 1 . The theoretical values were calculated via the Stokes-Einstein equation for the translational diffusion coefficient and via the Stokes-Einstein-Debye equation for the rotational diffusional coefficient. The relative error for the latter is 5\% for DMSO/glycerol and 19\% for Triton $\mathrm{X}-100$. In the following section, we briefly discuss several possible origins of the error. Firstly, the mismatch of the

Table 1 Comparison between experimental and theoretical values of translational and rotational diffusion coefficients

\begin{tabular}{lllll}
\hline & \multicolumn{2}{l}{$D_{\mathrm{t}}\left(\mu \mathrm{m}^{2} \mathrm{~s}^{-1}\right)$} & & $D_{\mathrm{r}}\left(\mathrm{s}^{-1}\right)$ \\
\cline { 2 - 3 } \cline { 5 - 6 } Solvent & Theo. & Exp. & Theo. & Exp. \\
\hline Glycerol/DMSO & $1.99 \times 10^{-3}$ & $2.60 \times 10^{-3}$ & $6.74 \times 10^{-3}$ & $7.04 \times 10^{-3}$ \\
Triton X-100 & $1.38 \times 10^{-3}$ & $1.56 \times 10^{-3}$ & $4.70 \times 10^{-3}$ & $3.83 \times 10^{-3}$
\end{tabular}

refractive indices between the silica core and poly(MPTS) and the dispersion media and the point spread function are known to induce a distortion of axial distance along the $z$ axis, ${ }^{30}$ which induced an error in the determination of the vector of the optical axis. In addition to performing a correction based on either experimental measurements or theoretical considerations, a simple solution would be using the same materials for both the core and the shell by a synthetic route, which is also expected to be easily index-matched by the solvents. Secondly, photo-bleaching of the used fluorescent markers is also a known problem for long-time imaging. The resulting decrease in the signal/noise ratio can increase the error in determining the center of brightness. The third origin of error could be from the polydispersity of the model colloids, as it is close to $6 \%$ in our case. This can be improved by improving the monodispersity of the colloids.

\section{Conclusions}

In summary, we report a confocal microscopy method for concurrently tracking the translational and rotational dynamics of colloidal spheres in three dimensions. As a proof-of-concept, we devised and synthesized bicolor colloidal spheres with an eccentric core-shell structure and measured their translational and rotational dynamics in two different dilute systems. We found the measured values to reasonably agree with the theoretical values which were calculated via the Stokes-Einstein and Stokes-EinsteinDebye equations. Because this method is based on confocal microscopy, it can be applied in 3D to concentrated colloidal systems, soft materials systems (such as gel, glass, etc.) and complex bio-systems.

\section{Acknowledgements}

We thank Prof. Jiajia Zhou in Beihang University for helpful discussion and critical reading of the manuscript.

\section{References}

1 W. B. Russel, D. A. Saville and W. R. Schowalter, Colloidal Dispersions, Cambridge University Press, 1999.

2 S. Jabbari-Farouji, G. H. Wegdam and D. Bonn, Phys. Rev. E: Stat., Nonlinear, Soft Matter Phys., 2012, 86, 041401.

3 Y. Peng, L. Lai, Y.-S. Tai, K. Zhang, X. Xu and X. Cheng, Phys. Rev. Lett., 2016, 116, 068303.

4 K. Adachi, R. Yasuda, H. Noji, H. Itoh, Y. Harada, M. Yoshida and K. Kinosita, Jr., Proc. Natl. Acad. Sci. U. S. A., 2000, 97, 7243-7247.

5 T. G. Mason and D. A. Weitz, Phys. Rev. Lett., 1995, 74, 1250-1253.

6 Z. Cheng and T. G. Mason, Phys. Rev. Lett., 2003, 90, 018304.

7 V. Degiorgio, R. Piazza and R. B. Jones, Phys. Rev. E: Stat. Phys., Plasmas, Fluids, Relat. Interdiscip. Top., 1995, 52, 2707-2717.

8 S. A. Rogers, M. Lisicki, B. Cichocki, J. K. G. Dhont and P. R. Lang, Phys. Rev. Lett., 2012, 109, 098305. 
9 J. Kanetakis, A. Tölle and H. Sillescu, Phys. Rev. E: Stat. Phys., Plasmas, Fluids, Relat. Interdiscip. Top., 1997, 55, 3006-3014.

10 M. P. Lettinga, G. H. Koenderink, B. W. M. Kuipers, E. Bessels and A. P. Philipse, J. Chem. Phys., 2004, 120, 4517-4529.

11 L. Sanchez, P. Patton, S. M. Anthony, Y. Yi and Y. Yu, Soft Matter, 2015, 11, 5346-5352.

12 J. C. Crocker and D. G. Grier, J. Colloid Interface Sci., 1996, 179, 298-310.

13 A. Mohraz and M. J. Solomon, Langmuir, 2005, 21, 5298-5306.

14 B. Liu, T. H. Besseling, M. Hermes, A. F. Demirörs, A. Imhof and A. van Blaaderen, Nat. Commun., 2014, 5, 3092.

15 F. C. Cheong and D. G. Grier, Opt. Express, 2010, 18, 6555-6562.

16 A. Wang, T. G. Dimiduk, J. Fung, S. Razavi, I. Kretzschmar, K. Chaudhary and V. N. Manoharan, J. Quant. Spectrosc. Radiat. Transfer, 2014, 146, 499-509.

17 J. Behrend, J. N. Anker, B. H. McNaughton, M. Brasuel, M. A. Philbert and R. Kopelman, J. Phys. Chem. B, 2004, 108, 10408-10414.

18 S. M. Anthony, L. Hong, M. Kim and S. Granick, Langmuir, 2006, 22, 9812-9815.
19 D. Dinsmore, E. R. Weeks, V. Prasad, A. C. Levitt and D. A. Weitz, Appl. Opt., 2001, 40, 4152-4159.

20 P. Tan, N. Xu and L. Xu, Nat. Phys., 2014, 10, 73-79.

21 A. van Blaaderen and P. Wiltzius, Science, 1995, 270, 1177-1179.

22 U. Gasser, E. R. Weeks, A. Schofield, P. N. Pusey and D. A. Weitz, Science, 2001, 292, 258-262.

23 E. R. Weeks, J. C. Crocker, A. C. Levitt, A. Schofield and D. A. Weitz, Science, 1999, 287, 627-630.

24 A. Yethiraj and A. van Blaaderen, Nature, 2003, 421, 513-517. 25 S. Jiang, J. Yan, J. K. Whitmer, S. M. Anthony, E. Luijten and S. Granick, Phys. Rev. Lett., 2014, 112, 218301.

26 K. V. Edmond, M. T. Elsesser, G. L. Hunter, D. J. Pine and E. R. Weeks, Proc. Natl. Acad. Sci. U. S. A., 2012, 109, 17891-17896.

27 N. A. M. Verhaegh and A. van Blaaderen, Langmuir, 1994, 10, 1427-1438.

28 S. Sacanna, M. Korpics, K. Rodriguez, L. Colón-Meléndez, S.-H. Kim, D. J. Pine and G.-R. Yi, Nat. Commun., 2013, 4, 1688.

29 T. Savin and P. S. Doyle, Biophys. J., 2005, 88, 623-638.

30 A. Diaspro, F. Federici and M. Robello, Appl. Opt., 2002, 41, 685-690. 